\title{
Classifying Surface Points Based on Developability Using Machine Learning
}

\author{
Vahide Bulut \\ İzmir Katip Çelebi University, Departmant of Engineering Sciences, İzmir, Turkey, (ORCID: 0000-0002-0786-8860), vahide.bulut@ikcu.edu.tr
}

(International Conference on Design, Research and Development (RDCONF) 2021 - 15-18 December 2021)

(DOI: 10.31590/ejosat.1039296)

ATIF/REFERENCE: Bulut, V. (2021). Classifying Surface Points Based on Developability Using Machine Learning. European Journal of Science and Technology, (32), 171-176.

\begin{abstract}
The classifiers K-nearest neighbor (KNN), Multiclass support vector machine (MSVM), Decision Tree (DT), Discriminate Analysis (DA), Naive Bayes (NB), Random Forest (RF), and Ensemble Tree (ET) are the most well-known methods in machine learning. They are used in many fields like pattern recognition, medical disease analysis, user smartphone classification, text classification, etc. This paper presents a new framework for 3D surface point type classification using the most known classification methods in machine learning and the principal curvatures, the binormal vector, the cosine value of the angle between the normal vector and binormal vectors. The purpose of this study is to classify data points according to their developability. Also, the comparison between these methods is given to measure developability based on the accuracy and the processing time using several 3D surface examples.
\end{abstract}

Keywords: Machine learning, classification, principal curvatures, binormal vector.

\section{Makine Öğrenmesini Kullanarak Açılabilirliğe Dayalı Yüzey Noktalarını Sınıflandırma}

$\ddot{O} \mathbf{z}$

Sınıflandırıcılar K-en yakın komşu (KNN), Çok sınıflı destek vektör makinesi (MSVM), Karar Ağacı (DT), Ayrım Analizi (DA), Naive Bayes (NB), Rastgele Orman (RF) ve Topluluk Ağacı (ET) makine öğrenmesinde en iyi bilinen yöntemlerdir ve örüntü tanıma, tıbbi hastalık analizi, kullanıcı akıllı telefon sınıflandırması, metin sınıflandırması gibi birçok alanda kullanılmaktadır. Bu makale, makine öğrenmesinde en bilinen sınıflandırma yöntemlerini ve asal eğrilikleri, binormal vektörü, normal vektör ve binormal vektörler arasındaki açının kosinüs değerini kullanarak 3B yüzey noktası tipi sınıflandırması için yeni bir çerçeve sunmaktadır. Bu çalışmanın amacı, veri noktalarını açılabilirliklerine göre sınıflandırmaktır. Ayrıca, bu yöntemler arasındaki karşılaştırma, çeşitli 3B yüzey örneği kullanılarak doğruluk ve işleme süresine dayalı olarak açılabilirliği ölçmek için verilmiştir.

Anahtar Kelimeler: Makine öğrenmesi, sınıflandırma, asal eğrilikler, binormal vektör. 


\section{Introduction}

Surface normals, principal directions, and principal curvatures are utilized in 3D computer vision to find solutions for some basic tasks, such as segmentation, surface classification, surface reconstruction, and registration Krsek et al. (1998). We can use the principal curvatures to determine the characterization of a surface due to the principal curvatures' invariant properties at a surface point. Many researchers studied the Gaussian and the mean curvatures on several kinds of surfaces such as faces and human bodies.

Shape recognition from range data is studied in terms of the principal curvatures in Vemuri et al. (1986). On the other hand, the principal curvatures are utilized for object recognition in Deng et al. (2007). Darveau et al. expressed the effect of the principal curvatures 3D rendering of relatively noisy ultrasound angiograms without degrading the spatial resolution Darveau et al. (2018). Shape similarity is measured in Wang et al. (2018) in terms of the principal curvatures.

A 3D object classification method is presented based on the numerical surface point signatures on interest points of $3 \mathrm{D}$ objects point cloud Rimkus et al.(2014). A 3D surface object classification method based on shape description and suitable pattern classification techniques is proposed in Shen et al. (2004). They used normalization and the spherical harmonic parameterization techniques to define a surface shape and derive a dual high dimensional landmark representation. Zheng et al. presented a new deep learning method to classify surface materials according to the acceleration signal and a corresponding surface texture image using Fully Convolutional Network (FCN) Zheng, et al. (2016).

This paper provides a new perspective for $3 \mathrm{D}$ surface point type classification regarding developability using the most utilized classification methods in machine learning and the principal curvatures, the binormal vector of S. Frenet frame, and the angle between the surface normal, and the binormal vectors. Additionally, we compare these methods to determine the developability of surface data points in terms of accuracy and the processing time using different 3D surface examples.

The rest of the paper is given in this manner: Section 2 provides basic information about a surface and the most popular machine learning classification methods. The proposed method is given in Section 3, while Section 4 presents several experimental studies and their results. Finally, Section 5 concludes the study.

\section{Preliminaries}

This section briefly describes the curvatures of a surface and classification methods in machine learning.

\subsection{Curvature of a Surface}

A surface is a subset of $\mathbb{R}^{3}$ and looks like a piece of $\mathbb{R}^{2}$ in the vicinity of any given point. The following definitions describe the notion of a surface in $\mathbb{R}^{3}$.
Definition 1: If a surface patch $\boldsymbol{S P}: U \rightarrow \mathbb{R}^{3}$ is smooth, and the vectors $\boldsymbol{S} \mathbf{P}_{\mathbf{u}}$ and $\boldsymbol{S} \mathbf{P}_{\mathbf{v}}$ are linearly independent at all points $(u, v) \in$ $U$, this surface is called a regular surface.

A normal unit vector is defined at a point $\boldsymbol{P}$ on this surface as

$$
\mathbf{n}_{\mathbf{S P}}=\frac{\mathbf{S P}_{\mathbf{u}} \times \mathbf{S} \mathbf{P}_{v}}{\left\|\mathbf{S P}_{\mathbf{u}} \times \mathbf{S} \mathbf{P}_{v}\right\|}
$$

Definition 2: Suppose that $\mathbf{S P}(u, v)$ is a surface patch of a surface $\mathbf{S}$ and $\mathbf{r}(t)=\mathbf{S P}(u(t), v(t))$ is a unit speed curve in $\boldsymbol{S P}$. The normal curvature of this curve is expressed with the following equation:

$$
\kappa_{n}=L \mathrm{u}^{\prime 2}+2 M \mathrm{u}^{\prime} \mathrm{v}^{\prime}+N \mathrm{v}^{\prime 2}
$$

in which $L, M$ and $N$ are coefficients of the second fundamental form and defined by

$$
L=\mathbf{S P}_{\mathbf{u u}} \mathbf{n}, \quad M=\mathbf{S P}_{\mathbf{u v}} \cdot \mathbf{n} \text {, and } \quad N=\mathbf{S P}_{\mathbf{v v}} \cdot \mathbf{n} .
$$

Proposition 1: The principal curvatures $\kappa_{n 1}$ and $\kappa_{n 2}$ are the maximum and minimum of the normal curvature in (2) at the point $\boldsymbol{P}$. The principal curvatures can be written in the mean and Gaussian curvatures by Pressley, A. (2010).

$$
K=\kappa_{n 1} \cdot \kappa_{n 2} \text { and } \quad H=\frac{\kappa_{n 1}+\kappa_{n 2}}{2} .
$$

or in a quadratic equation form

$$
\kappa_{n}^{2}-2 H \kappa_{n}+K=0
$$

which has solutions as

$$
\left\{\begin{array}{l}
\kappa_{n 1}=H+\sqrt{H^{2}-K} \\
\kappa_{n 2}=H-\sqrt{H^{2}-K} .
\end{array}\right.
$$

The principal and Gaussian curvatures $\kappa_{n 1}, \kappa_{n 2}$ and $K$ have great importance in determining the shape of the surface. The classification of points on a surface in terms of the principal curvatures is presented in Table 1 .

Table 1: Classification of surface points based on principal curvatures.

\begin{tabular}{|c|c|c|c|}
\hline & $\kappa_{n 1}<0$ & $\kappa_{n 1}=0$ & $\kappa_{n 1}>0$ \\
\hline$\kappa_{n 2}<0$ & Concave Ellipsoid & Concave Cylinder & Hyperboloid Surface \\
\hline$\kappa_{n 2}=0$ & Concave Cylinder & Plane & Convex Cylinder \\
\hline$\kappa_{n 2}>0$ & Hyperboloid Surface & Convex Cylinder & Convex Ellipsoid \\
\hline
\end{tabular}

Definition 3: Let $\boldsymbol{r}(t)$ be a non-unit speed curve. S. Frenet frame of this curve is:

$$
\frac{\boldsymbol{r}^{\prime}(t)}{\left\|\boldsymbol{r}^{\prime}(t)\right\|}=\boldsymbol{T}, \quad \frac{\boldsymbol{r}^{\prime} \times \boldsymbol{r}^{\prime \prime}}{\left\|\boldsymbol{r}^{\prime} \times \boldsymbol{r}^{\prime \prime}\right\|} \times \boldsymbol{T}=\boldsymbol{N} \text { and } \quad \frac{\boldsymbol{r}^{\prime} \times \boldsymbol{r}^{\prime \prime}}{\left\|\boldsymbol{r}^{\prime} \times \boldsymbol{r}^{\prime \prime}\right\|}=\boldsymbol{B}
$$

in which $\boldsymbol{T}, \boldsymbol{N}$, and $\boldsymbol{B}$ are the unit tangent vector, principal normal vector, and binormal vector, respectively Kreyszig, E. (1959). 


\subsection{Classification Methods in Machine Learning}

Machine learning is a branch of computer science and tries to obtain meaning from data. We describe objects around us according to their features (or attributes). Intuitively, a featured class is composed of similar objects. A training process is to state the connection between the output and the input features Patil and Kulkarni, (2019). Features can be determined in the form of names, categories, types of entities, etc. On the other hand, nonnumerical features can be converted to numerical values. There are some basic classification methods in machine learning.

The k-nearest neighbor classifier ( $\mathrm{k}-\mathrm{NN})$ is used widely since it is theoretically elegant and simple to use Duda et al. (2001). This method classifies an input in data by retrieving the $\mathrm{k}$ nearest prototypes from the labeled reference set.

The decision tree algorithm classifies the given data by containing the minimum number of nodes Sulaiman, M. A. (2020). It is related to knowledge of relationships that include nodes and connections Priyanka and Kumar, (2020).

An SVM model is applied to binary classification by dividing data elements either in 1 or 0 . However, the same principle MSVM is

\section{Proposed Method}

The principal curvatures have been used for object recognition. Therefore this study constructs a bridge between the object recognition and surface points types based on machine learning.

Assume that data points belonging to a surface are given. First, the binormal vectors, the normal vectors, and the angles between these vectors are estimated. Next, the principal curvatures are calculated at each point in the data. Moreover, some well-known classification algorithms in machine learning are used to state the best method that classifies the points without knowing the principal curvatures. The Gaussian curvature is utilized to determine the points which have developability properties. Because a surface that has zero Gaussian curvature is called a developable surface. Finally, we apply machine learning classification methods K-nearest neighbor (KNN), Multiclass support vector machine (MSVM), Decision Tree (DT), Discriminate Analysis (DA), Naive Bayes (NB), Random Forest (RF), and Ensemble Tree (ET), and obtain accuracy with the computational cost for each one, respectively. The proposed algorithm is presented in Algorithm 1. utilized, and the multiclass problem is broken down into multiple binary classification cases Ahuja and Yadav, (2012).

For the discriminant analysis method, it generates classes based on the Gaussian distributions. The fitting function calculates the parameters for each class, and the trained classifier seeks the class for new data according to the smallest misclassification cost Tharwat, A. (2016).

A Naive Bayes classifier method is based on applying Bayes' theorem, so it is a simple probabilistic classifier. It assumes any class feature as independent from any other feature Berrar, D. (2018).

Like the KNN and the decision tree methods, a random forest method is also a tree-based method, and this method is based on a random vector's sampled values for each tree independently with the same distribution Breiman, L. (2001).

On the other hand, ensemble methods construct a classifier set and classify new data points by taking their predictions' vote Dietterich T.G. (2000).

Algorithm 1: The proposed method

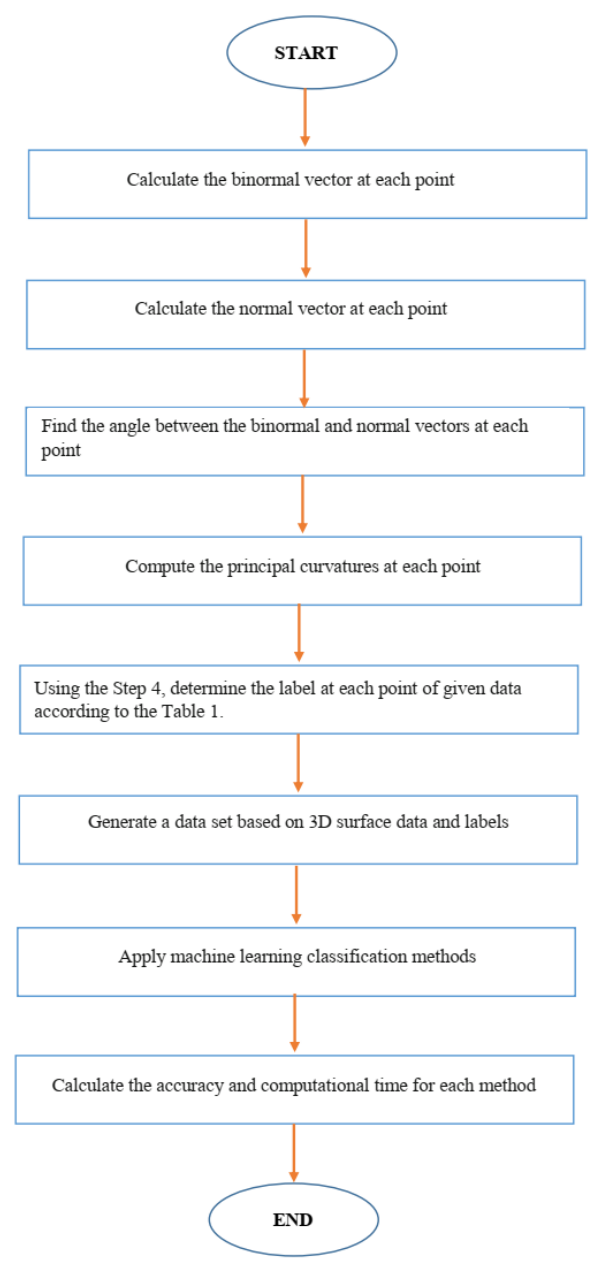




\section{Experimental Study and Results}

In this section, as shown in Table 2, five surface examples are considered to apply the most known classification methods in machine learning. Next, the comparision between these methods is presented in Table 3 regarding the accuracy and processing time (s).

Table 2. Example Surfaces

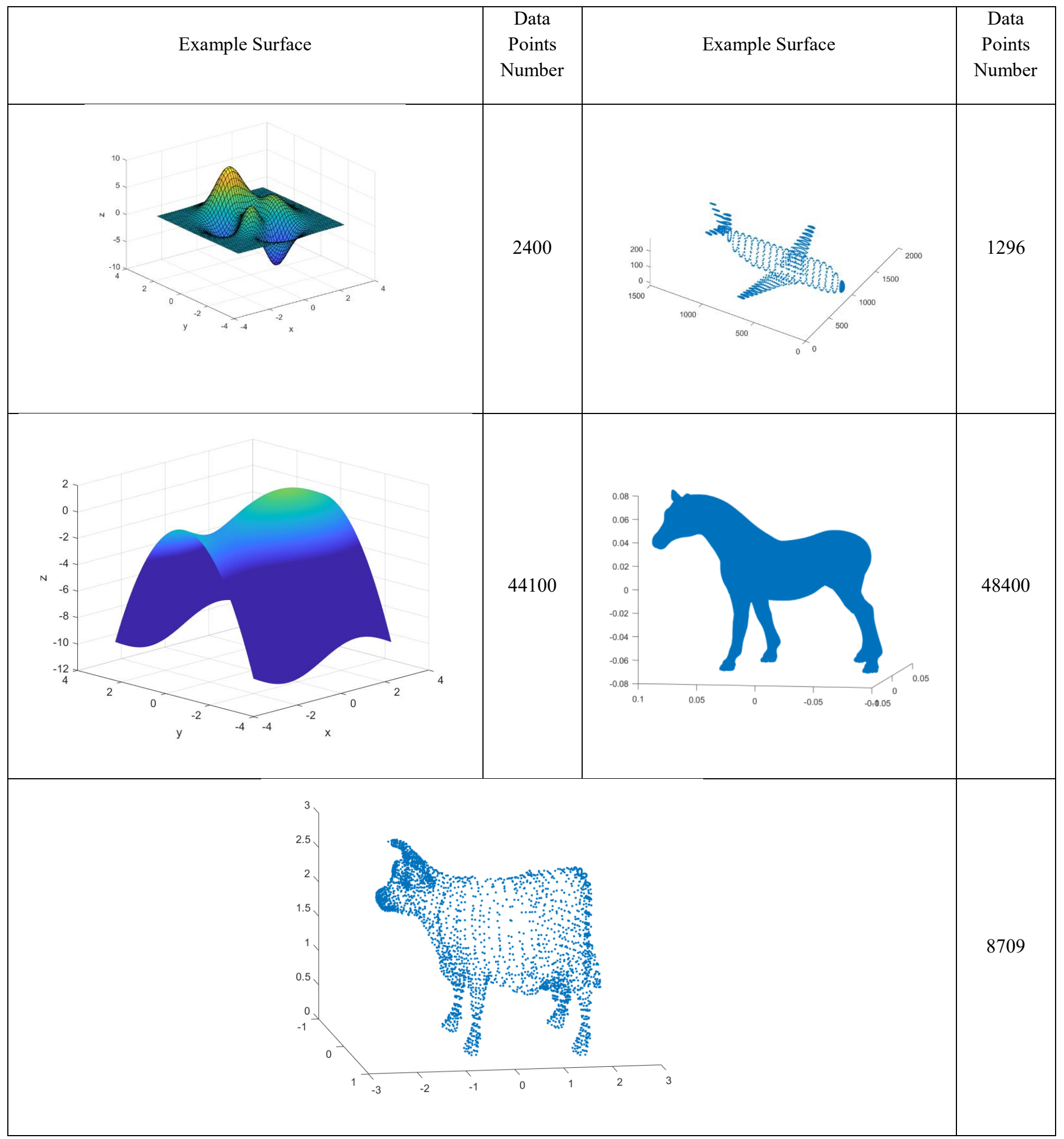


Table 3. Comparing of Classification Methods for Surface Points Types

\begin{tabular}{|c|c|c|c|c|c|c|c|c|c|c|c|}
\hline \multicolumn{2}{|c|}{ Data Sets } & \multicolumn{2}{|c|}{$\# 1$} & \multicolumn{2}{|c|}{$\# 2$} & \multicolumn{2}{|c|}{$\# \mathbf{3}$} & \multicolumn{2}{|c|}{$\# 4$} & \multicolumn{2}{|c|}{$\# 5$} \\
\hline & & Acc.(\%) & Time(s) & Acc.(\%) & Time(s) & Acc.(\%) & Time(s) & Acc.(\%) & Time(s) & Acc. $(\%)$ & Time(s) \\
\hline \multirow{7}{*}{ 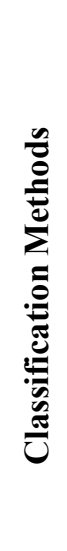 } & KNN & 96.9175 & 1.1279 & 65.8092 & 0.4183 & 99.941 & 4.5401 & 67.8347 & 4.5796 & 60.6905 & 1.1358 \\
\hline & MSVM & 96.5278 & 0.4993 & 68.5567 & 0.1844 & 99.9169 & 1.6232 & 68.5468 & 56.3616 & 61.7577 & 0.5853 \\
\hline & DT & 95.6944 & 0.0208 & 68.8185 & 0.6755 & 100 & 3.7776 & 64.6901 & 15.1990 & 59.812 & 1.1621 \\
\hline & DA & 90.0048 & 0.4884 & 63.6599 & 0.5279 & 97.6916 & 1.0110 & 58.7128 & 0.9953 & 57.5299 & 0.5125 \\
\hline & NB & 83.4652 & 0.7025 & 63.274 & 1.7716 & 49.9773 & 0.7592 & 49.3905 & 1.5157 & 57.6368 & 0.7445 \\
\hline & $\mathbf{R F}$ & 97.0427 & 5.8494 & 75.6274 & 6.5809 & 100 & 36.2251 & 78.2107 & 191.5423 & 65.1418 & 15.6860 \\
\hline & ET & 95.4604 & 23.7250 & 72.0697 & 18.9153 & 100 & 230.3312 & 66.5847 & 397.1837 & 59.9858 & 28.2437 \\
\hline
\end{tabular}

As seen in Table 3, the random forest (RF) method is the most accurate one among the applied methods. The RF method makes classification providing almost $100 \%$ accuracy for general 3D

surfaces \#1 and \#3, while providing accuracies in the range $65 \%$ $80 \%$ for the surfaces with specific shapes. However, the processing time for the $\mathrm{RF}$ is the second most taking time to calculate the proper classes. On the other hand, the Ensemble Tree (ET) method is the one that has the most spending time even though it cannot find the accuracy with high percentages.

\section{Conclusion}

In this study, we utilized the most-known machine learning classification methods K-nearest neighbor (KNN), Multiclass support vector machine (MSVM), Decision Tree (DT), Discriminate Analysis (DA), Naive Bayes (NB), Random Forest $(\mathrm{RF})$, and Ensemble Tree (ET) to classify $3 \mathrm{D}$ surface points in terms of developability. The classification is made according to the principal curvatures, binormal vector, and the angle between the normal and binormal vector features. We compared the mentioned methods using several 3D examples in terms of accuracy and computational time. Table 2 expresses that the RF method is the best classifier for surface point developability even if it takes some time to process. Also, we realized that it is more accurate for general 3D surfaces such as \#1 and \#3 than object surfaces.

\section{References}

Krsek, P., Lukacs, G. and Martin, R. R. (1998). Algorithms for computing curvatures from range data. In In The Mathematics of Surfaces VIII, Information Geometers, pages $1-16$.
Vemuri, B. C., Mitiche, A. and Aggarwal, J. K. (1986). Curvature based representation of objects from range data. Image and Vision Computing, 4:107-14.

Deng, H., Zhang, W., Mortensen, E., Dietterich, T. and Shapiro, L. (2007) Principal curvature-based region detector for object recognition. In 2007 IEEE Conference on Computer Vision and Pattern Recognition, pages 1-8.

Tremblay-Darveau, C., Sheeran, P. S., Vu, C. K., Williams, R., Bruce, M. and Burns, P. N. (2018). 3-d perfusion imaging using principal curvature detection rendering. IEEE Transactions on Ultrasonics, Ferroelectrics, and Frequency Control, 65(12):2286-2295.

Wang, F., You, H.J., Qiu, X.L. and Yao, X.H. (2018). Shape similarity measure method based on principal curvature enhancement distance transformation. Journal of Infrared and Millimeter Waves, 37(1).

Rimkus, K., Lipnickas, A., \& Sinkevicius, S. (2014). Classification of 3D Point Cloud Using Numerical Surface Signatures on Interest Points. Elektronika Ir Elektrotechnika, 20, 8-11.

Shen, L., Ford, J.C., Makedon, F., \& Saykin, A.J. (2004). A surface-based approach for classification of 3D neuroanatomic structures. Intell. Data Anal., 8, 519-542.

Zheng, H., Fang, L., Ji, M., Strese, M., Özer, Y. and Steinbach, E. (2016). "Deep Learning for Surface Material Classification Using Haptic and Visual Information," in IEEE Transactions on Multimedia, vol. 18, no. 12, pp. 2407-2416.

Pressley, A. (2010). Elementary Differential Geometry. SpringerVerlag London.

Kreyszig, E. (1991). Differential Geometry. Dover Publications, Inc., New York.

Patil, S. and Kulkarni, U. (2019). Accuracy Prediction for Distributed Decision Tree using Machine Learning approach, in 2019 3rd International Conference on Trends in Electronics and Informatics (ICOEI), pp. 1365-1371.

Duda, R. O., Hart, P. E. and Stork, D. G. (2001). Pattern Classification, 2nd edition. John Wiley \& Sons, New York. 
Sulaiman, M. A. (2020). Evaluating Data Mining Classification Methods Performance in Internet of Things Applications, Journal of Soft Computing and Data Mining, vol. 1, no. 2, pp. $11-25$.

Ahmed, O. and Brifcani, A. (2019). Gene Expression Classification Based on Deep Learning, in 2019 4th Scientific International Conference Najaf (SICN), Al-Najef, Iraq, pp. $145-149$.

Patil, D. V. and Bichkar, R. S. (2006). A Hybrid Evolutionary Approach To Construct Optimal Decision Trees With Large Data Sets, in 2006 IEEE International Conference on Industrial Technology, pp. 429-433.

Priyanka and Kumar, D. (2020). Decision tree classifier: a detailed survey," International Journal of Information and Decision Sciences, vol. 12, no. 3, pp. 246-269.

Ahuja, Y., \& Yadav, S.K. (2012). Multiclass Classification and Support Vector Machine By Yashima Ahuja \& Yadav.

Tharwat, Alaa. (2016). Linear vs. quadratic discriminant analysis classifier: a tutorial. International Journal of Applied Pattern Recognition. 3. 145. 10.1504/IJAPR.2016.079050.

Berrar, Daniel. (2018). Bayes' Theorem and Naive Bayes Classifier. 10.1016/B978-0-12-809633-8.20473-1.

Breiman, L. (2001). Random Forests. Machine Learning 45, 532.

Dietterich T.G. (2000). Ensemble Methods in Machine Learning. In: Multiple Classifier Systems. MCS 2000. Lecture Notes in Computer Science, vol 1857. Springer, Berlin, Heidelberg. 